\title{
NOTE ON A PAPER BY SHEPPERD ON THE BRAID GROUP
}

\author{
SEYMOUR LIPSCHUTZ ${ }^{1}$
}

1. Introduction. Let $F_{n}=\left\langle x_{1}, x_{2}, \cdots, x_{n}\right\rangle$ be the free group on which the braid group of $n$ strings, $B_{n}$, acts as a group of automorphisms. If $\alpha$ is a braid in the subgroup $A_{n}$ of $B_{n}$ where

$$
B_{n} / A_{n} \cong \Sigma_{n}=\text { symmetric group of order } n ! \text {, }
$$

that is, the subgroup with identity permutation, then (see [1]), as an automorphism of $F_{n}$,

$$
\begin{aligned}
\alpha\left(x_{i}\right) & =T_{i}\left(x_{\lambda}\right) x_{i} T_{i}\left(x_{\lambda}\right)^{-1} \quad(i=1,2, \cdots, n), \\
\alpha\left(x_{1} x_{2} \cdots x_{n}\right) & =x_{1} x_{2} \cdots x_{n} .
\end{aligned}
$$

Let $Q_{n}$ be the subgroup of $A_{n}$ which is generated by the braids

$$
R_{i}=\left(\sigma_{1} \sigma_{2} \cdots \sigma_{i-2}\right)^{i-1}\left(\sigma_{i} \sigma_{i+1} \cdots \sigma_{n}\right)^{i-n} \quad(i=2, \cdots, n+1),
$$

where the $\sigma_{i}$ are the usual generators of $B_{n}$. (See $[1 ; 4]$.)

A textile manufacturer asked the following question: Can one decide whether or not a braid in $A_{n}$ is also in $Q_{n}$ ? (It is possible to "weave" a braid in $Q_{n}$ while keeping its ends "tied together.") Shepperd [6] solved the above decision problem using the generators and relations of the braid group and subgroups. In this paper we give another distinct solution working in terms of automorphisms of free groups. In particular, we use formulas developed in $[3 ; 4]$.

The author wishes to thank his teacher, and friend, Professor Wilhelm Magnus who has contributed more than anyone else towards the author's knowledge of mathematics.

2. The algorithm. To decide whether or not $\alpha$ in $A_{n}$ is also in $Q_{n}$, we present the following:

Algorithm. Rewrite $\alpha$ as the automorphism

$$
\alpha\left(b_{i}\right)=W_{i}\left(b_{\lambda}\right) \quad(i=1,2, \cdots, n),
$$

where $b_{i}=x_{i} x_{i+1} \cdots x_{n}$. Set $b_{1}=I$, the identity element, in (1) and freely reduce. Since the group $B_{1}$ generated by $b_{1}$ is characteristic under $A_{n}, \alpha$ now induces an automorphism $\alpha^{*}$ of the free group

Received by the editors March 8, 1962.

${ }^{1}$ Work on this paper was partially sponsored by a grant from the National Science Foundation, NSF-G9659 during the summer of 1961 at the Courant Institute of Mathematics. 
$F_{n-1}^{*}=F_{n} / B_{1}$. We have two possibilities:

(a) The automorphism $\alpha^{*}$ is not of the form

(2) $\alpha^{*}\left(b_{i}\right)=V\left(b_{2}, \cdots, b_{n}\right) b_{i} V\left(b_{2}, \cdots, b_{n}\right)^{-1} \quad(i=2, \cdots, n)$,

that is, an inner automorphism of $F_{n-1}^{*}$. Then $\alpha$ is not in $Q_{n}$.

(b) The automorphism $\alpha^{*}$ is of the form (2). We now see if

$$
b_{1}^{t} \alpha\left(b_{i}\right) b_{1}^{-t}=V\left(R_{2}, \cdots, R_{n}\right)\left(b_{i}\right) \quad(i=1, \cdots, n),
$$

for any $t$ where $|t|<T$. Here $T$ is equal to the maximum length of $\alpha\left(b_{i}\right)$ plus the maximum length of $V\left(R_{2}, \cdots, R_{n}\right)\left(b_{i}\right)$. (Notice that $V\left(R_{2}, \cdots, R_{n}\right)$ is an automorphism acting on $\left.b_{i \cdot}\right)$

CASE (a). If there is no $t$ for which (3) is true then $\alpha$ is not in $Q_{n}$. CASE (b). If there is a $t$ for which (3) is true then $\alpha$ is in $Q_{n}$, in fact,

$$
\alpha=R_{n+1}^{t} V\left(R_{2}, \cdots, R_{n}\right) .
$$

THEOREM. The above algorithm is a solution to the decision problem of whether a braid in $A_{n}$ is also in $Q_{n}$.

Proof. As was shown in $[3 ; 4]$, the braids $R_{i}$ are the following automorphisms of $F_{n}$ :

$$
\begin{aligned}
R_{i}\left(b_{j}\right) & =b_{i} a_{i j}^{-1} b_{j} b_{i}^{-1} a_{i j} \cdots(i=2, \cdots, n), \\
R_{n+1}\left(b_{j}\right) & =b_{1} b_{j} b_{1}^{-1}
\end{aligned}
$$

where

$$
a_{i j}= \begin{cases}b_{1} & \text { if } j<i, \\ I, \text { the identity, } & \text { if } i \leqq j .\end{cases}
$$

It is known [5] that $R_{n+1}$ is in the center of $B_{n}$ and that the other $R_{i}$ are free generators of a free subgroup. Therefore, for any $\beta$ in $Q_{n}$,

$$
\beta=R_{n+1}^{t} W\left(R_{2}, \cdots, R_{n}\right) .
$$

But, by (4) and (5), the induced automorphism of $\beta$ acting on $F_{n-1}^{*}$ is

$$
\beta^{*}\left(b_{i}\right)=W\left(b_{2}, \cdots, b_{n}\right) b_{i} W\left(b_{2}, \cdots, b_{n}\right)^{-1} \quad(i=2, \cdots, n),
$$

an inner automorphism. (Another verification that the $R_{i}, i \neq n+1$, are free.) Thus a necessary condition for $\alpha$ in $A_{n}$ to be in $Q_{n}$ is for $\alpha^{*}$ to be an inner automorphism of $F_{n-1}^{*}$. Further, if (2) is true then, by (4) and (5),

$$
\alpha \equiv V\left(R_{2}, \cdots, R_{n}\right) \bmod B_{1}
$$


Now, by (5) and (6), $\alpha$ is in $Q_{n}$ if and only if (3) is true for some $t$. A simple cancellation argument in (3) will show that we need not consider $t$ if $|t|>T$.

Thus the algorithm is a solution to the above decision problem.

\section{BIBLIOGRAPHY}

1. E. Artin, Theory of braids, Ann. of Math. (2) 48 (1947), 101-126.

2. - Theorie der Zöpfe, Abh. Math. Sem. Univ. Hamburg 4 (1925), 47-72.

3. S. Lipschutz, On the braid group, Ph.D. Dissertation, New York University, N. Y., 1960.

4. - On a finite matrix representation of the braid group, Arch. Math. 12 (1961), 7-12.

5. W. Magnus, Über Automorphismen von Fundamentalgruppen berandeter Flächen, Math. Ann. 105 (1931), 52-74.

6. J. A. H. Shepperd, Braids which can be plaited with their threads tied together at each end, Proc. Roy. Soc. Ser. A 265 (1962), 229-244.

New YoRK UNIVERSITY AND

Polytechnic Institute of Brooklyn 